\title{
Germanica
}

\section{Metaphern für den Menschen}

Die Entwicklung der anthropologischen Metaphorologie Hans

Blumenbergs

\section{David Adams}

\section{OpenEdition}

\section{Journals}

Édition électronique

URL : http://journals.openedition.org/germanica/2442

DOI : $10.4000 /$ germanica.2442

ISSN : 2107-0784

\section{Éditeur}

Université de Lille

\section{Édition imprimée}

Date de publication : 31 décembre 1990

Pagination : 171-191

ISBN : 9782913857025

ISSN : 0984-2632

\section{Référence électronique}

David Adams, « Metaphern für den Menschen », Germanica [Online], 8 | 1990, Online erschienen am: 28 November 2014, abgerufen am 06 Oktober 2020. URL : http://journals.openedition.org/germanica/ 2442 ; DOI : https://doi.org/10.4000/germanica.2442

Ce document a été généré automatiquement le 6 octobre 2020.

(ㄷ) Tous droits réservés 


\title{
Metaphern für den Menschen
}

Die Entwicklung der anthropologischen Metaphorologie Hans

Blumenbergs $^{1}$

\author{
David Adams
}

Alle diese Gleichnisse wollen eigentlich nur sagen, daß das Ungreifbare ungreifbar ist, und das haben wir gewußt.

Franz Kafka

1 Seit 45 Jahren ist Hans Blumenberg mit der Entwicklung seiner Philosophie beschäftigt, und er ist heute produktiver als je zuvor. Aus diesem Grund könnte eine Einschätzung seiner Metaphorologie möglicherweise noch zu früh kommen, aber er hat uns bislang kaum einen Anlaß gegeben, der eine fundamentale Änderung seiner Position erwarten ließe. Bislang ist er vielmehr in der Entfaltung und in der Ausarbeitung seiner Theorie überaus konsistent geblieben. Jedenfalls müssen wir über seinen zukünftigen Weg nicht spekulieren. Wir können das Stück, das er schon zurückgelegt hat, verfolgen, ohne weiter darüber nachzudenken, wo er «ankommen» wird. Um zu Beginn einen Kontext für das Veständnis seiner Leistung bereitzustellen, gehe ich zunächst kurz auf die Disputation zwischen Ernst Cassirer und Martin Heidegger ein, die 1929 in Davos (Schweiz) stattgefunden hat. In dieser Davoser Disputation, die in Gestalt eines Protokolls erhalten ist, das Assistenten angefertigt haben ${ }^{2}$, kommt deutlich zum Ausdruck, daß die beiden Philosophen in einer Vielzahl miteinander verbundener Themen konträre Positionen vertreten. Sie vermittelt einen Eindruck von der bedrohlichen Herausforderung, die die «Metaphysik» (Heidegger) für die «philosophische Anthropologie» (Cassirer) darstellte, ehe Blumenberg sich der letzteren annahm.

\section{Cassirer und Heidegger in Davos}

$2 \mathrm{Zu}$ den meisten Fragen nehmen Cassirer und Heidegger entgegengesetzte Standpunkte ein. Der eine hat die nach vorn schauende Einstellung der Aufklärung und interpretiert 
die Geschichte der Kultur als Fortschritt vom Mythos zum Logos; der andere ist ein durch und durch romantischer Denker, der immer zurückblickt zum Ursprung der Sprache, der Kultur, der Geschichte. An einer Stelle der Disputation faßt Heidegger die wesentliche Differenz ihrer Orientierungen treffend zusammen: «Der terminus a quo bei Cassirer ist vollkommen problematisch. Meine Position ist umgekehrt: Der terminus a quo ist meine zentrale Problematik, die ich entwickle. Die Frage ist: Ist der terminus ad quem bei mir so klar ? (260) Heideggers Vorwurf ist also, daß der, der sich auf die Produkte der Kultur konzentriert, Schwierigkeiten hat, ihren Ursprung zu erklären. Er scheint aber zu erkennen, daß diese Behauptung leicht umkehrbar ist: Vielleicht hat der, der die Ursprünge entdecken will, Schwierigkeiten mit den Produkten ${ }^{3}$.

Die Debatte über die Termini oder Grenzpunkte, zwischen denen die Kultur sich entfaltet, ist eng verbunden mit der Frage nach der Totalität und dem Absoluten. Beide Philosophen versuchen, die Beziehung des Menschen zu Phänomenen, die über seine Erfahrung hinausgehen, verständlich $\mathrm{zu}$ machen. Was verbindet ihn mit transzendenten, unbedingten oder absoluten Wahrheiten? Wie kommt ein endliches Wesen dazu, die Ewigkeit zu erahnen ? Nach Cassirers Behauptung geht der menschliche Weg zum Unendlichen «durch das Medium der Form», einer vom Menschen selbst geschaffenen Form. «Die Unendlichkeit», so erklärt er, «ist gerade die Totalität, die vollkommene Erfüllung der Endlichkeit selbst. Aber diese Erfüllung der Endlichkeit konstituiert eben die Unendlichkeit. Goethe: "Willst du ins Unendliehe schreiten, geh' nur im Endlichen nach allen Seiten» (258). Für Heidegger dagegen hat «der Mensch als endliches Wesen» nur «eine gewisse Unendlichkeit im Ontologischen. Der Mensch ist nie unendlich und absolut im Schaffen des Seienden selbst, sondern er ist unendlich im Sinne des Verstehens des Seins» (252). Und dieses Seinsverständnis gibt es nach Heidegger vor aller Kultur und Kulturphilosophie aufgrund der Zeitlichkeit des Daseins: «Im Wesen der Zeit» nämlich, behauptet Heidegger, liegt «eine innere Transzendenz» (254).

4 Trotz und wegen der unterschiedlichen Terminologie beider Philosophen ist ihre radikale Differenz in der Auffassung des Unendlichen deutlich erkennbar. Für Heidegger trifft man es am terminus a quo, der zeitlichen Struktur des Daseins, aller Kultur zuvor; Cassirer identifiziert es mit dem terminus ad quem, dem Höhepunkt der Kultur. Im Diskussionsprotokoll wird klar, daß Cassirer bereit war, die unversöhnlichen Meinungsverschiedenheiten hinzunehmen, wohingegen Heidegger es auf einen endgültigen Sieg abgesehen hatte. Cassirers Annahme einer Mannigfaltigkeit symbolischer Formen - «das Sein, das von einer Mannigfaltigkeit von funktionellen Bestimmungen und Bedeutungen ausgeht» (266) - machte ihn wohl eher dazu geneigt, auch abweichende Meinungen gelten zu lassen. Heidegger dagegen, entschlossen hinter die Mannigfaltigkeit von Seinsstrukturen zurückzugehen, konnte es sich nicht leisten, so «ökumenisch» zu sein. Sein Wille, die Philosophie auf die «zentrale Frage der Metaphysik» zu beschränken - auf die Frage nach dem Sinn von Sein -, hat seine dogmatische Ablehnung der philosophischen Anthropologie als «viel zu eng, viel zu vorläufig» zur Folge. Und sein Angebot kam an: Allgemein herrschte Übereinstimmung darüber, daß Heidegger in Davos die Oberhand gewonnen hatte. So leistete die Disputation ihren Beitrag zum Verfall des Neukantianismus.

5 Das Werk Cassirers aber hat sich in einem von Heidegger unbeabsichtigten Sinn als «vorläufig» erwiesen: Blumenberg hat die philosophische Anthropologie erweitert, unterstützt und verwandelt, und dies zum Teil auch, um sie gegen die Attacken 
Heideggers widerstandsfähig $\mathrm{zu}$ machen. Das erneute Interesse an der Davoser Disputation und am Neukantianismus deutet an, daß der Fall Cassirer mittlerweile zu einer Revision ansteht; aber schon lange vorher hat Blumenberg der philosophischen Anthropologie ihre Legitimität zurückzugeben versucht. Dabei hat er sowohl Elemente aus dem Werk Cassirers als auch aus dem Werk Heideggers in seine eigene Philosophie integriert. Wie Cassirer interessiert er sich für die Funktion symbolischer Formen, aber er hat Cassirers philosophische Anthropologie so umgewendet, daß sie, wie Heidegger, auch auf den terminus a quo zurückblickt. Diese Parallele zu Heidegger darf aber nicht überbewertet werden: denn für Blumenberg ist der Ursprung unzugänglich und abweisend. Beide, Cassirer wie Heidegger, wollen sich einem der Grenzpunkte annähern. Blumenberg aber konzentriert sich auf die Bewegung, die von ihnen wegführen soll.

6 Aber bevor Blumenbergs Philosophie sich so weit entwickeln konnte, hatte sie mit dem schwierigen Problem des Absoluten zu kämpfen. Dieser Kampf dauert zwar noch bis heute an, aber den ersten großen Schritt ihn durchzustehen und hinter sich zu bringen, hat Blumenberg mit der Veröffentlichung der Paradigmen $z u$ einer Metaphorologie (1960) vollzogen ${ }^{4}$. In diesem frühen Werk neigte er noch dazu, wie Cassirer die Frage nach dem terminus a quo weitgehend zu vernachlässigen.

\section{Paradigma: Metapher}

«Was ist die Welt? «Welchen Anteil hat der Mensch am Ganzen der Wahrheit?» In den Paradigmen zu einer Metaphorologie sind dies Beispiele für «totale» Fragen, die Blumenberg in seinem Versuch, die Benennung oder Bestimmung des Absoluten zu vermeiden, gerade nicht $\mathrm{zu}$ beantworten sich bemüht. Blumenberg will dem Bedürfnis nach Antworten dieser Art widerstehen, indem er zeigt, daß sie ausnahmslos die Gestalt von Metaphern annehmen: Absolute Metaphern nämlich «repräsentieren das nie erfahrbare, nie übersehbare Ganze der Realität» $(20)^{5}$. An einen solchen begrifflichdirekten Zugang zum Ganzen der Realität zu glauben aber heißt, sich von Metaphern irreführen zu lassen. Die Schlußworte der frühen Abhandlung kündigen daher Blumenbergs Kampf gegen diese Irreführung an: «Die absolute Metapher, so sahen wir, springt in eine Leere ein, entwirft sich hier auf der tabula rasa des theoretisch Unerfüllbaren; hier hat sie die Stelle des nicht mehr lebendigen absoluten Willens eingenommen. Metaphysik erwies sich uns oft als beim Wort genommene Metaphorik; der Schwund der Metaphysik ruft die Metaphorik wieder an ihren Platz» (142).

8 Nichtsdestotrotz illustriert Blumenbergs Aufsatz unbemerkt die Schwierigkeit der Absage an die Illusion transzendenter Erkenntnis. Zur Verdeutlichung dieser Schwierigkeit zunächst eine kurze Passage über unser Bedürfnis nach einer solchen Erkenntnis: «Absolute Metaphern "beantworten" jene vermeintlich naiven, prinzipiell unbeant wortbar en Fragen, deren Relevanz ganz einfach darin liegt, daß sie nicht eliminierbar sind, weil wir sie nicht stellen, sondern als im Daseinsgrund gestellte vorfinden» (19). Dies ist der Grundgedanke, auf den Blumenberg seine philosophische Anthropologie aufbaut. Aber der zu diesem Zeitpunkt noch kaum entwickelte Zustand seiner Anthropologie bringt Probleme mit sich, die ihn in einen Widerspruch verwickeln. In dem Augenblick nämlich, in dem Blumenberg die Frage nach dem terminus a quo der Metapher stellt, liefert er als Antwort genau die Art von absoluter Erkenntnis, der er gerade zu entsagen versucht: er bestimmt unsere Wißbegierde, 
unser Fragebedürfnis als einen invarianten, int er subjektiven Aspekt menschlicher Existenz - als etwas, das jenseits aller kulturellen Prozesse liegt ${ }^{6}$. Dieser Konflikt zwischen Absicht und Durchführung hat Auswirkungen auf das Verhältnis zwischen Vernunft und Rhetorik, wie es sich in den Paradigmen zeigt. In der Einleitung kritisiert Blumenberg die Erwartung, die Descartes im Discours de la Méthode formuliert: daß es einen Endzustand der Philosophie geben könne, in dem alles in klaren, deutlichen und unveränderlichen Begriffen definiert sein würde. Dagegen setzt Blumenberg die anticartesische Auffassung der Geschichte, die in seiner Konzeption der absoluten Metapher zum Ausdruck kommt - wir haben gesehen, daß solche Metaphern einen Typus von Erkenntnis liefern, der resistent ist gegen eine Übersetzung in eindeutige Terminologie. Zwar bilden Metaphern «die Substruktur des Denkens, ... den Untergrund, die Nährlösung der systematischen Kristallisationen». Es handelt sich um eine «katalysatorische Sphäre, an der sich zwar ständig die Begriffswelt bereichert, aber ohne diesen fundierenden Bestand dazu umzuwandeln und aufzuzehren» (10).

Wenn Blumenberg aber den Begriff der absoluten Metapher benutzt, um diesen Typ der Erkenntnis abzugrenzen und einzuschränken, so offenbart dies, noch optimistisch, ein Zutrauen zu und ein Interesse an der Fähigkeit der Vernunft, weit und weiter in den Bereich der Rherotik einzudringen. Er betont ausdrücklich die Unangemessenheit des Gebrauchs von Metaphern für sein eigenes Projekt: «Als Metaphorologie Betreibende haben wir uns schon der Möglichkeit beraubt, in Metaphern "Antworten" auf jene unbeant wortbar en Fragen zu finden» (19).

Die Abhandlung Blumenbergs besteht aus Geschichten von absoluten Metaphern, die so sorgfältig ausgewählt worden sind, daß sie als Paradigmata dienen können. Die so entstandene «Typologie von Metapherngeschichten» (84) soll den Bereich der Metapher abstecken, um ihn deutlich von anderen Weisen des Weltzugangs $\mathrm{zu}$ unterscheiden: vom rationalen (logischen), vom mythischen, vom symbolischen. Sogar der Titel des Werks - zu einer Metaphorologie - scheint die cartesische Erwartung einer Steigerung der Rationalität menschlichen Verhaltens zu teilen: als Weg zu einer Wissenschaft (logos) der Metapher.

Eine Metapher, «Paradigma», taucht nun allerdings häufig in den Paradigmen auf, ohne selber zum Gegenstand der Untersuchung zu werden ${ }^{7}$. Es ist gerade diese linguistische Metapher, die es Blumenberg erlaubt, die gesamte Bandbreite jener Formen zu durchmessen, in denen Menschen sich zum Absoluten in Beziehung setzen; diese Metapher gibt den anderen Metaphern in der Abhandlung Struktur und Einheit. Daß sie diese Leistung erbringt, ist aber genau das, was sie selber «absolut» macht. Die Vollständigkeit der Typologie ist so ein sicheres Zeichen dafür, daß die Typologie nicht vollständig ist: eine Metapher - Paradigma - ist zwangsläufig entschlüpft, um so den Boden zu liefern, auf dem die Typologie aufgebaut ist. Der Zweck der Paradigmen ist es, zur Herrschaft der Vernunft zu führen, und doch ist diese Vernunft, will sie ihre Herrschaft ausüben, abhängig von Rhetorik: von der Paradigma-Metapher. Paradigmen $z u$ einer Metaphorologie ist ein Titel, der deutlich macht, wie Rhetorik sich immer wieder gerade dann am stärksten zur Geltung bringt, wenn die Vernunft ihre Autonomie zu festigen und auszubauen sucht. Blumenbergs implizite Abwertung der Metapher gegenüber dem Begriff geht zurück auf eine Geschichtsauffassung, nach der die Wissenschaft, der Logos, den Mythos ersetzt; und diese Auffassung wiederum ist die Folge einer Vernachlässigung dessen, was beide Bereiche gemeinsam haben: den terminus a quo (Wir finden später den gleichen Vorwurf gegen Cassirer gewendet in 
Arbeit am Mythos, 186). Obwohl Blumenberg in seinem frühen Werk die Metapher bereits von ihrer Funktion her begreift, wird sein Verständnis dieser Funktion erst später, nämlich im Zusammenhang seiner ausführlicheren Beschäftigung mit ihrem terminus a quo, weiter vertieft.

Die Widersprüche in der Paradigmen-Abhandlung weisen auf die Schwierigkeit, wenn nicht Unmöglichkeit seines ambitiösen Projektes hin: Es bigt Bedürfnisse, die auf bestimmte Arten von Erkenntnis ausgerichtet sind und die unbefriedigt zu lassen gar nicht so leicht ist. Erst später gesteht er zu, daß immer dann, wenn eine Metapher verworfen wird, sie eine Frage hinterläßt, «auf die dann eine neue Antwort fällig wird, wenn und weil es nicht gelingt, die Frage selbst kritisch zu destruieren und am System der Welterklärung Amputationen vorzunehmen» (Legitimität 43). Mag sein, daß eine Philosophie der symbolischen Formen kein innerliches Bedürfnis danach hat, den terminus a quo näher zu bestimmen; aber wenn eine «Umbesetzung», in der eine Antwort, eine Philosophie oder eine Epoche an die Stelle einer anderen tritt, sich durchsetzt und Bestätigung findet, so sind dies «rhetorische Akte» (Anthropologische Annäherung 129). Und eine Philosophie, die Fragen unbeantwortet läßt, wird in der Regel weniger überzeugend sein als ihre Vorläufer und Konkurrenten. Dies zeigte sich in Davos, wo Heidegger durch eine bessere Erklärung des terminus a quo gegenüber Cassirer die Oberhand gewann. Ein Eingehen auf die Frage nach dem terminus a quo ist notwendig, um die Überzeugungskraft einer philosophischen Position zu vergrößern. Vielleicht erklärt dies, warum Blumenberg, obwohl er in seinem frühen Werk absichtlich bestimmte Fragen meidet, sie trotzdem unbemerkt beantwortet: mit «Begriffen» wie «Daseinsgrund» und «Paradigma».

\section{Ein anthropologischer terminus a quo}

13 In den nächsten zwanzig Jahren wird sich Blumenberg in zunehmendem Maße der Frage nach dem terminus a quo bewußt und er sinnt auf Antworten für sie. In den Paradigmen zu einer Metaphorologie bestimmt er, wie wir gesehen haben, den terminus a quo in einem einzigen Satz: Die Metapher entspringt aus den im Daseinsgrund immer schon gestellten Fragen. In der Legitimität der Neuzeit (1966; 1973/1976) ${ }^{8}$ zeigt er dann aber, daß diese Fragen nicht konstant, sondern so geschichtlich sind, wie die Antworten auf sie. Das Buch verteidigt die Neuzeit gegen den Angriff der Säkularisierungsthese, in der behauptet wird, daß das neuzeitliche Denken (z.B. der Fortschrittsgedanke) aus nichts anderem besteht als dem verkleideten und zu Unrecht angeeigneten Gedankengut des Mittelalters (z.B. der christlichen Eschatologie). Blumenberg behauptet dagegen, daß es zwar eine die beiden Epochen übergreifende Identität gibt; diese ist allerdings nicht eine «Identität der Sache», sondern «der Funktion». Neuzeitliche Metaphern nämlich, obgleich in «Substanz» oder «Inhalt» von ihren theologischen Vorläufern verschieden, können dennoch diesen ähnlich erscheinen, aber nur deshalb, weil sie gezwungen sind, auf dieselben Fragen zu antworten: «Dieses System [der Welt-und Selbstdeutung des Menschen] hat die christliche Theologie in unserer geistigen Geschichte en scheidend und vor allem im Sinne der Ausweitung bestimmt; sie hat neue "Stellen" im Rahmen der über die Welt und den Menschen für möglich gehaltenen Aussagen geschaffen, die nicht wieder "eingespart" oder in theoretischer Ökonomie unbesetzt gehalten werden konnten. Unbeantwortbare Fragen nach der Totalität der Welt und der Geschichte, nach der 
Herkunft und Bestimmung des Menschen brauchte es für die Theologie nicht zu geben, und darin gründet die Leichtigkeit, mit der sie Titel in den Haushalt der menschlichen Wissensbedürfnisse eingebracht hat, deren Einlösung jeder nicht gleicherweise auf transzendente Quellen sich berufenden Erkenntnis schwer oder gar unmöglich werden mußte» (41 f.).

Diese Passage soll zeigen, daß die von der christlichen Theologie im «Daseinsgrund» verankerten Fragen genau dieselben sind wie die, die durch absolute Metaphern beantwortet werden. Indem die philosophische Anthropologie Blumenbergs sich hier nur auf die «Titel» konzentriert, die erst vor verhältnismäßig kurzer Zeit dem «Haushalt der menschlichen Wissensbedürfnisse» hinzugefügt wurden, liefert sie hier zwar eine partielle Erklärung des terminus a quo, meidet aber immer noch das Absolute und entgeht so der Versuchung zur Totalisierung des Wissens.

Impliziert allerdings ist in dieser Darstellung der Glaube, daß der Mensch immer im Besitz von Fragen oder Bedürfnissen ist, die auf den terminus a quo der Kulturentwicklung zielen. Diese Implikation führt Blumenberg erst in seinem Aufsatz Anthropologische Annäherung an die Aktualität der Rhetorik (1971) weiter aus. Bis heute haben die Werke Blumenbergs sich Themen zugewandt - der Genesis der Neuzeit, der Metapher, dem Mythos -, bei deren Behandlung seine anthropologische Grundposition im Hintergrund geblieben ist. Die Anthropologische Annäherung an die Rhetorik aber ist die einzige Arbeit, die ausdrücklich dem Thema gewidmet ist, das uns hier beschäftigt. In diesem Aufsatz führt Blumenberg für das Tier mit dem wachsenden und schrumpfenden Haushalt der Wissensbedürfnisse einen besonderen Namen ein: Mängelwesen. Der Ausdruck stammt von A. Gehlen und aus einer Tradition der Sprachphilosophie, die von Herder ihren Ausgang nimmt. Im Kontext von Blumenbergs eigener Entwicklung aber muß er als Nachfolger des Begriffs des Daseinsgrundes aus der Paradigmen-Abandlung betrachtet werden. Blumenberg betrachtet die Rhetorik nun als das notwendige Werkzeug eines Wesens, das einen Mangel an sinnhafter Einfügung in eine Umwelt kompensieren muß. Anders als den Tieren nämlich fehlen dem Menschen die Instinkte, die aus-reichen würden, sein Verhalten in allen Situationen festzulegen; und Rhetorik ist eines der Mittel, die ihm dabei helfen, die Krisen, denen er so ausgesetzt ist, lebend zu überstehen. «Der Mangel des Menschen an spezifischen Dispositionen zu reaktivem Verhalten gegenüber der Wirklichkeit, seine Instinktarmut also, ist der Ausgangspunkt für die anthropologische Zentralfrage, wie dieses Wesen trotz seiner biologischen Indisposition zu existieren vermag. Die Antwort läßt sich auf die Formel bringen: indem es sich nicht unmittelbar mit dieser Wirklichkeit einläßt. Der menschliche Zugang zur Wirklichkeit ist indirekt, umständlich, verzögert, selektiv, und vor allem "metaphorisch"» (115).

Hier bringt sich der Metaphorologe nicht mehr so schnell um die Möglichkeit, Antworten in Metaphern zu finden. Die Gefahr eines solchen Verzichtes erwähnt Blumenberg in seinen Bemerkungen über «Identität» gegen Ende des Aufsatzes: «Die anthropologischen Zugänge zur Rhetorik [konvergieren] auf eine zentrale deskriptive Feststellung: der Mensch hat zu sich selbst kein unmittelbares, kein rein "innerliches" Verhältnis. Sein Selbstverständnis hat die Struktur der "Selbstäußerlichkeit"... Der Mensch begreift sich nur über das, was er nicht ist, hinweg. Nicht erst seine Situation, sondern schon seine Konstitution ist potentiell metaphorisch» (134 f.). Wenn wir den Menschen, wie Blumenberg es vorschlägt, als ein Wesen verstehen, für das die Welt keinen vorherbestimmten und festgelegten Sinn hat, dann erscheint Heideggers Plan 
absurd: ernst zu machen mit der Frage nach dem Sinn von Sein würde uns nicht dazu führen, auf der Suche nach dem Ursprung die Tradition abzuwerfen.

Mit der Entwicklung dieses «ontologischen» terminus a quo im Begriff des Mängelwesens ebnet sich Blumenberg den Weg zu einer vollständigeren Konzeption des historischen terminus a quo. In Arbeit am Mythos (1979) fügt er diesen entscheidenden Baustein seiner philosophischen Anthropologie hinzu. Genauer gesagt, führt er nun den Begriff des «Absolutismus der Wirklichkeit» ein: den bedrohlichen terminus a quo, den das Mängelwesen mittels Mythos und Metapher auf Distanz halten muß $ß^{9}$. Den «Absolutismus der Wirklichkeit» charakterisiert er als die Situation, in der «der Mensch die Bedingungen seiner Existenz annähernd nicht in der Hand hatte und, was wichtiger ist, schlechthin nicht in seiner Hand glaubte» (9). Der Mythos ist nun das Mittel, durch das der Mensch auf die Herausforderung dieser Situation antwortet und die mächtige Gewalt des Fremden zerlegt. Eine solche «Gewaltenteilung» ermöglicht es dem Menschen, auf die überwältigende Unbestimmtheit und Vieldeutigkeit der Welt stückweise $\mathrm{zu}$ reagieren. Beide Begriffe, «Absolutismus der Wirklichkeit» und «Mängelwesen», beschreiben einen terminus a quo, der abweisend ist: «Zu dem Anfang, auf den konvergiert, wovon hier die Rede ist, will nichts zurück. Alles bemißt sich vielmehr in Distanz zu ihm» (28).

Daher die Erkenntnis, die in der Paradigmen-Abhandlung zu fehlen schien: «daß die Antithese von Mythos und Vernunft eine späte und schlechte Erfindung ist, weil sie darauf verzichtet, die Funktion des Mythos bei der Überwindung jener archaischen Fremdheit der Welt selbst als eine vernünftige anzusehen» (56). Wie die Metapher und die «Antworten» der Neuzeit ist auch der Mythos von seiner Funktion her bestimmt. In Arbeit am Mythos greift Blumenberg aber weiter in die Geschichte zurück und tiefer in das Mängelwesen hinein, um das Minimum an Identität nicht mehr nur für zwei Epochen, sondern nun für die gesamte Geschichte der Kultur zu finden ${ }^{10}$. Indem er untersucht, welches die Funktion ist, die alle symbolischen Formen teilen, gelingt ihm eine Extrapolation zurück zu ihrem gemeinsamen Ursprung.

Die Leistung, die die symbolischen Formen zu erbringen haben, ist niemals abgeschlossen; der Mensch wird niemals einen «Absolutismus der Bilder und Wünsche» genießen können, der durch die «Suprematie des Subjekts» charakterisiert ist. Gerade das ständige Schwanken zwischen den Absolutismen - zwischen Wirklichkeit und Phantasie, Hilflosigkeit und Verfügungsmacht, Frage und Antwort, Unbestimmtheit und Sinnfülle -: das ist nichts anderes als das, was wir "Geschichte» nennen ${ }^{11}$. Zur Erhaltung dieser Bewegung trägt Blumenberg bei, indem er sich vorzugsweise auf die Entfernung von statt auf die Annäherung an diese Extreme konzentriert. Wer sich zwischen ihnen aufhalten will, muß sich davor hüten, an einem der beiden Grenzwerte anzukommen und dort zu verharren.

\section{Metaphorische Anthropologie}

Die Entwicklung eines anthropologischen terminus a quo für die Metaphorologie fällt bei Blumenberg mit der wachsenden Einsicht in die metaphorische Natur der philosophischen Anthropologie zusammen. Blumenbergs früherer Versuch, Antworten auf Fragen zu vermeiden, die sich auf das nicht Erlebbare und nicht Erfahrbare richten, hat nun den Platz für eine imaginative Spekulation freigemacht, die weit über das hinausgeht, was erlebt und erfahren wird und werden kann. In Arbeit am Mythos zum 
Beispiel steigert er die rhetorische Kraft, die dem Begriff des «Absolutismus der Wirklichkeit» zu eigen ist, indem er ihn noch mit einem narrativen Inhalt füllt: «Wie immer das vormenschliche Wesen ausgesehen haben mag, das durch einen erzwungenen oder zufälligen Wechsel seines Lebensraumes veranlaßt wurde, den sensorischen Vorteil der Selbstaufrichtung zum bipeden Gang wahrzunehmen und gegen alle internen Nachteile der organischen Funktion zu stabilisieren - es hatte in jedem Fall den Schutz einer verborgeneren und angepaßteren Lebensform verlassen, um sich den Risiken des erweiterten Horizonts seiner Wahrnehmung als denen seiner Wahrnehmbarkeit auszusetzen... Es war ein Situationssprung, der den unbesetzten Fernhorizont zur dauernden Gewärtigung des bis dahin Unbekannten machte. Was in der Verdindung des Übergangs vom schrumpfenden Regenwald auf die Savanne mit der Besiedelung von Höhlen entstand, war die gleichzeitige Bewältigung neuer Leistungsanforderungen beim Nahrungserwerb außerhalb der Werkstätten mit dem alten Vorteil ungestörter Fortpflanzung und Aufzucht des lange lernbedürftigen Nachwuchses, jetzt im Schutz leicht abzuschirmender Gehäuse (10).

«Ist eine größere Spekulation vorstellbar» - dies ist die nur zu verständliche Reaktion eines Kritikers auf diese Passage, der bemerkt, daß «Blumenberg trotz seiner andauernden Ablehnung der Suche nach dem Ursprung diese Suche selber nicht aufgibt $»^{12}$. Aber Blumenbergs Suche ist nicht so sehr auf einen Ursprung an sich gerichtet als vielmehr auf eine Theorie dieses Ursprungs, die unser Bedürfnis nach solchen Geschichten befriedigt ohne dem, was wir an einsichtig begründetem Wissen besitzen, zu widersprechen. So macht die Arbeit am Mythos auf ihr eigenes rhetorisches Fundament aufmerksam, indem sie vom «Absolutismus der Wirklichkeit» als von einem «Grenzbegriff» spricht, der erreicht wird kraft einer Extrapolation zurück ins «Archaische» oder in die «Vorvergangenheit», zurück also, heißt das, in ein ganz unzugängliches Gebiet ${ }^{13}$.

Die Selbstreflexion, mit der diese Extrapolation durchgeführt wird, transformiert die Widersprüche und «toten» Metaphern der Paradigmen-Abhandlung in wirkungsvolle Paradoxien und lebendige Metaphern. Nun erkennt Blumenberg, daß «Übertragungen... geleistet, aber nicht beim Wort genommen werden» müssen (Arbeit am Mythos, 13). Seine philosophische Anthropologie beantwortet damit Fragen, die beides zugleich sind: unbeantwortbar und unvermeidbar. Die Metaphorik dieser Anthropologie ist notwendig und notwendigerweise «vorläufig», und in ihrer ausgereiften Gestalt nimmt sie Rücksicht auf beide Notwendigkeiten, indem sie Antworten liefert, ohne sie beim Wort zu nehmen. Dieses Bewußtsein seiner eigenen Rhetorik schließt Form und Inhalt von Blumenbergs Werk zusammen und schafft eine Übereinstimmung zwischen seiner Behauptung, daß die Funktion der Ideen wichtiger ist als ihr Inhalt, und der Art und Weise, in der er diese Behauptung vorbringt.

Beobachtungen an Metaphern (1971) ist ein Nebenwerk, aber es zeigt Blumenberg bereits bei der Entwicklung dieser Selbstreflexion. Im vierten Abschnitt, «Paradigma, grammatisch», untersucht er «Paradigma» «als Metapher» (Indirekt wie immer, erwähnt er hier mit keinem Wort seine frühere Abhandlung, in der diese Metapher doch eine so wichtige Rolle spielt). «Beobachtungen an Metaphern» ist ein Titel, der die lockere Form des Aufsatzes reflektiert, die mit dem eher systematischen Zugang der Paradigmen-Abhandlung kontrastiert. Der Titel weist indes auch auf eines der zentralen Interessen des Aufsatzes hin: den Standpunkt des Beobachters der Metaphern zu lokalisieren, der selbst nie völlig «außerhalb» von Metaphern steht oder 
frei von ihnen ist. Ein Thema der Untersuchung ist hier ein topos, der den Beobachter zu lokalisieren hilft: die Metapher vom Schiffbruch mit Zuschauer.

Blumenbergs Interesse an der Schiffbruchmetapher hat ihren vollständigsten Ausdruck bisher in einem kleinen Buch gefunden: Schiffbruch mit Zuschauer. Paradigma einer Daseinsmetapher (1979). Es verfolgt die Geschichte dieser Metapher von der Antike bis zur Gegenwart. Jetzt spricht Blumenberg ausdrücklich von einer «Daseinsmetapher» statt vom «Daseinsgrund», eine Entwicklung, die er im Rhetorik-Aufsatz schon vorweggenommen hat, wenn er dort die Konstitution des Menschen als eine potentiell metaphorische beschreibt. Die Schiffbruchmetapher scheint ihr eigenes Schicksal und die Studie Blumenbergs darüber zu beschreiben: Er beobachtet ihren Zusammenbruch durch die Geschichte, in der ihre verschiedenen Bestandteile wegfallen - das Land, der Hafen, der Zuschauer und sogar das Schiff und sein Bruch. Die Geschichte der Metapher spitzt sich zu auf die Überlegung Jakob Burckhardts: «Wir möchten gerne die Welle kennen, auf welcher wir im Ozean treiben, allein wir sind diese Welle selbst» (66). Am Schluß stellt Blumenberg eine Frage, die das Buch schon knapp beantwortet hat: «Offenbar enthält das Meer noch anderes Material als das schon verbaute. Woher kann es kommen, um den neu Anfangenden Mut zu machen? Vielleicht aus früheren Schiffbrüchen?» (74). Blumenberg gibt der Metapher neues Leben, indem er sich selbst - und den Leser - zum Zuschauer des Schiffbruchs dieser Metapher macht. Innen und Außen, Subjekt und Objekt werden ununter scheidbar. Dies ist ein «Paradigma» für eine «Metaphorologie», die weiß und akzeptiert, daß Metaphern nicht nur Gegenstand der Analyse sind, sondern auch das Subjekt konstituieren, das diese Analyse durchführt. Indem er sich auf dem Meer der Metapher treiben läßt, vermeidet Blumenberg die Illusion, selber im Absoluten verankert zu sein.

In den achtziger Jahren wächst die Zahl der Metapherngeschichten Blumenbergs rapide. Durch alle diese Arbeiten hindurch zieht sich die Doppelbewegung, die wir schon in Schiffbruch fanden: hin zur Distanz und zur Unmittelbarkeit. Auf der einen Seite scheint jede Geschichte einer Metapher für sich vollständig und abgeschlossen zu sein: ein abgerundeter Gegenstand, der dem Historiker gegenübersteht. Auf der anderen Seite betont jede Arbeit das Ausmaß, in dem Beobachter und Beobachtungsakt selber metaphorisch, symbolisch sind, konstituiert durch eben die Metaphern, die gerade untersucht werden. Die Ambiguität der Position von Autor und Leser und die Vorliebe für den Begriff des Beobachters - ob als «Beobachter», "Theoretiker», «Zuschauer», «Anschauer» oder «Leser»" ${ }^{14}$ - zeigen die Selbstreflexion in Blumenbergs Versuchen, sein Verhältnis zur Vergangenheit zu beschreiben und herzustellen. Wie «neu» ist seine Anthropologie? Steht er schon mit einem Bein in der nächsten Epoche ? Teils innerhalb und teils außerhalb der Metaphern der Neuzeit ? Wie sehr kann und sollte man versuchen, auf den Übergang von einer Epoche zur anderen durch Änderung der Antworten und vielleicht sogar der Fragen Einfluß zu nehmen?

\section{Eine absichtlich vergessene Frage}

Blumenberg teilt - und steigert - die «ökumenische» Einstellung Cassirers. Wenn man erkennt, daß die Sprache auf Stellen und Prozesse hinweist, die sonst unsichtbar oder unwahrnehmbar wären oder überhaupt nicht existieren würden, dann wird es schwerer zu glauben, daß nur ein einziges Vokabular, eine einzige Interpretation oder ein einziges System der Erkenntnis das Recht hat, eine jede Situation zu bestimmen 
und zu beherrschen. Wenn man Metaphern nicht wörtlich nimmt, wird es schwerer, dogmatisch zu sein. Das bedeutet nicht, daß alle Antworten gleich gültig sind: jede muß äußerlich zu dem passen, was wir an einsichtig begründetem Wissen besitzen, und sie muß innerlich unsere früheren Erkenntniserwartungen befriedigen. Aber vermutlich können zwei oder mehr widersprüchliche Interpretationen gleich gut funktionieren. Die Begriffe «Absolutismus der Wirklichkeit» und «Mängelwesen» fügen sich in diese Auffassung ein, indem sie jeder Epoche und Ausdrucksweise - sogar denjenigen, die diesen Begriffen ausdrücklich widersprechen - eine fundamentale Legitimität zugestehen: gehören sie doch hinein in den stets fortlaufenden Prozeß der Selbsterhaltung. Natürlich gibt es auch andere Systeme der Interpretation, die mit Blumenbergs «ökumenischem» konkurrieren können, und die ihre eigenen Gründe für die Legitimität einer philosophischen Anthropologie haben ${ }^{15}$.

oft brigt Blumenberg diesen radikalen ökumenischen Gedanken dadurch zum Ausdruck, daß er geltend macht, die Produkte jeder Kultur, jeder Epoche, jedes Individuums seien bewahrenswert und erhaltungswürdig, wie obsolet sie gegenwärtig auch erscheinen mögen. Er spricht von «der elementaren Obligation, Menschliches nicht verloren zu geben» (Cassirers gedenkend 170 und passim), oder besteht darauf, daß «denkwürdig ist, was Menschen je gedacht haben» (Die Lesbarkeit der Welt 409). Und die Pflicht, sich zu erinnern, erstreckt sich auch auf die Erinnerung an die Fragen: «Die Philosophie vertritt nur einen allgemeineren Befund an jeder Kultur: den der Ununterdrückbarkeit ihrer elementaren Bedürfnisse und Fragen durch deren vermeintliche Überwindung. Kultur ist auch Respektierung der Fragen, die wir nicht beantworten können» (Nachdenklichkeit 61). Immer wenn ein Stück der Geschichte der Vergessenheit anheimfällt, verengt sich unser Verständnis dessen, was es bedeutet, ein Mensch zu sein, und ineins damit auch der Spielraum unserer Möglichkeiten ${ }^{16}$. Ökumenisch zu sein trägt somit selbst zum Fortgang der Selbsterhaltung bei. Trotzdem ist der Rat Goethes, den Cassirer zitiert, nicht leicht zu befolgen: wie können wir «nach allen Seiten» im Endlichen gehen? Offensichtlich können wir nicht alle vergangenen geistigen Formationen und gedanklichen Bildungen in gleichem Maße lebendig erhalten, sondern müssen ihnen verschiedene Grade von Bedeutsamkeit zumessen. Selbsterhaltung heißt also auch, selektiv sein zu müssen. Wie sehr auch immer eine Person (oder Kultur) der ökumenischen Einstellung zuneigen mag: sie kann nicht allen Fragen und Antworten in gleichem Maße und mit gleicher Energie entgegenkommen. Und auch ob und wie wir an den Fragen, die unsere Aufmerksamkeit verlangen, etwas ändern können, bleibt problematisch. Nehmen wir zum Beispiel die Frage nach dem Subjekt der Geschichte. Diese «Stelle» wurde in der Neuzeit «um-besetzt», vom Gott auf den Menschen übertragen: «Die kühnste Metapher, die die größte Spannung zu umfassen suchte, hat daher vielleicht am meisten für die Selbstkonzeption des Menschen geleistet: indem et den Gott als das Ganz-Andere von sich absolut hinwegzudenken versuchte, begann er unaufhaltsam den schwierigsten rhetorischen Akt, nämlich den, sich mit diesem Gott zu vergleichen» (Anthropologische Annäherung 135).

28 Wie ich schon angedeutet habe, ist Blumenbergs Einstellung gegenüber der Neuzeit zwiespältig: Wiederholt hat er hingewiesen auf die Exzesse der neuzeitlichen Konzeption des Menschen, wie «legitim» jene Umbesetzung auch gewesen sein mag ${ }^{17}$. Inwieweit findet er aber selber eine Antwort, die diese Leerstelle ausfüllen könnte? 
Gelingt es ihm, sie unbesetzt zu lassen, und so gleichsam eine «Amputation» der Frage vorzunehmen?

Das Bewußtsein der ästhetischen Natur eines bestimmten Typs von Antworten ist ein Zeichen dafür, daß die Fragen, die sie beantworten, an Dringlichkeit verloren haben. Die größere rhetorische Freiheit in der Behandlung des Absoluten, die Blumenberg sich seit den siebziger Jahren erlaubt, ist ein Anzeichen für seine wachsende Distanzierung von der Frage nach dem Absoluten. Die Frage nach dem Subjekt der Geschichte wird dann weniger dringend, wenn man erkannt hat, daß «der Singular von Geschichte selbst eine absolute Metapher» ist (Beodachtungen 168). In bezug auf diese Metapher ist die Metaphorologie Blumenbergs uneindeutig: Ist es so, daß die einzelnen Metapherngeschichten, die er veröffentlicht hat, zusammen eine einheitliche Geschichte konstituieren ? Daß er diese Frage im Hintergrund belassen hat, hat es ihm erlaubt, die einzelnen Geschichten zu entfalten. Sie umgrenzen und beschränken den Bereich der Metapher nicht, scheinen aber doch eine der Absichten der ParadigmenAbhandlung mit etwas anderen Mitteln zu realisieren: das Absolute rückt in größere Ferne und wird metaphorischer. Eine große Frage und der Versuch, sie kritisch zu de(kon)struieren, verdunkeln oft viele kleinere Fragen; und weder der Selbsterhaltung noch der ökumenischen Einstellung scheint besonders gut gedient zu sein, wenn die Frage nach dem Subjekt der Geschichte gestellt wird.

In dem Maße, in dem das Problem des Absoluten in den Hintergrund tritt, hat Blumenberg sich in andere verzweigte Gebiete vorgewagt. Im letzten Jahrzehnt hat er begonnen, auch andere literarische Gattungen auszuprobieren: Zeitungsaufsätze, «imaginäre Anekdoten», die von kleinen und abseitigen historischen Fakten ausgehen, Glossen, die Fabeln mehr adaptieren als kommentieren. Die Wende zu dieser offensichtlicher literarischen Arbeit hat ihren Niederschlag gefunden in Texten, die Blumenberg in der Frankfurter Allgemeinen Zeitung, in der Neuen Zürcher Zeitung und in den Akzenten veröffentlicht hat und auch in Gestalt kleinerer Bücher erschienen sind, in: Die Sorge geht über den Fluß (1987) und in der Matthäuspassion (1988). Diese Wende scheint denn auch zu einem Philosophen zu passen, der, wie ich gezeigt habe, sich der metaphorischen Struktur seines eigenen Denkens immer stärker bewußt wird. Aber diese eher literarischen Produkte bleiben änigmatisch; sie verwischen die Grenzen zwischen dem Imaginären und dem Historischen, dem Peripheren und dem Zentralen, dem Bedeutsamen und dem Unbedeutsamen. Vielleicht können sie auch als ein Versuch verstanden werden, einer großen Frage den Rücken zu kehren und auf der Basis dieser Abkehr vielen kleineren Fragen und Möglichkeiten, alten und neuen nachzugehen. Vielleicht versucht Blumenberg, sich selbst und uns abzulenken von dem, wovon wir glaubten, daß wir es wissen wollten. Vielleicht ist das, was er tut, eine Einübung in eine Erinnerung an die kleinen Fragen, die das Vergessen der großen Fragen lehrt. 


\section{NOTES}

1. - Der in englisch verfaßte Text dieses Essais wurde von Barbara Merker für seine Erstausgabe in Germanica 8/1990 ins Deutsche übersetzt.

2. - Der Text der Disputation ist kein «wortgetreues Protokoll»; J. Ritter (damals Cassirers Assistent) und O.F. Bollnow haben den Diskussionsverlauf anhand ihrer Notizen rekonstruiert. Die «Davoser Disputation zwischen Ernst Cassirer und Martin Heidegger» ist als Anhang enthalten in der vierten, erweiterten Auflage von M. Heidegger, Kant und das Problem der Metaphysik, Frankfurt/M, 1973, 246-268.

3. - Heideggers Selbstzweifel sind sehr berechtigt. Seine Konzeption des terminus ad quem ist keineswegs klar - oder viel zu klar. Dies wird besonders offensichtlich in einer vier Jahre später geäußerten Formulierung: «Der Anfang ist noch. Er liegt nicht hinter uns als das längst Gewesene, sondern er steht vor uns... Der Anfang ist in unsere Zukunft eingefallen, er steht dort als die ferne Verfügung über uns, seine Größe wieder einzuholen». Für einen, der das Ziel hat, zum Ursprung zurückzukehren, ist der terminus ad quem nicht länger mit den Produkten der Kultur gleichzusetzen; er ist identisch mit dem terminus a quo. Diese Einstellung, die für die Philosophie nicht unschädlich zu sein scheint - sie schließt zum Beispiel eine Philosophie der symbolischen Formen aus -, ist erst recht in einem ausdrücklich politischen Kontext beunruhigend: die zitierte Bemerkung findet sich nämlich in Heideggers berüchtigter Rektoratsrede, die er 1933 an der Universität Freiburg gehalten hat (Die Selbstbehauptung der deutschen Universität, Frankfurt/M 1983, 12 f.).

Während Heidegger den Nationalsozialismus schließlich als eine politische Bewegung beschreiben sollte, die ihre Möglichkeit einen neuen Anfang zu stiften, verriet, beschrieb ihn Cassirer getreu seiner aufklärerischen Geschichtsauffassung in Der Mythos des Staates als einen Anachronismus, als einen Rückfall auf eine primitivere Stufe der Geschichte.

Die Rede über die Aufgabe der Geisteswissenschaften an der Universität, die Hans Blumenberg 1961 in Gießen gehalten hat, kann als indirekte Antwort auf Heideggers Rede gelesen werden. Blumenberg geht dort davon aus, daß die «Geschichte ihre Wirklichkeit wesentlich darin hat, daß sie die Funktionen von ihren Ursprüngen und Innervationen trennt. "Geschichte" bedeutet, daß die im Ursprung waltenden Gründe nicht über das Werdende und schließlich Gewordene entscheiden. Sinn ist in der Geschichte keine Konstante... Die Geschichte kennt keine Wiederkehr... Es mag uns heute an positiven Formulierungen unserer Bildungsidee fehlen; aber dieses läßt sich doch sagen: Bildung ist ganz wesentlich Unverführbarkeit. Nach unserer eigenen geschichtlichen Erfahrung will es mir scheinen, daß das sehr viel und sehr positiv ist und daß wir sehr viel tun sollten, um es zu verwirklichen», (Weltbilder und Weltmodelle, in Nachrichten der Gießener Hochschulgesellschaft 30, 1960, 70, 73, 75).

4. - Alle in diesem Aufsatz zitierten Werke H. Blumenbergs sind, mit Ausnahme der folgenden, im Suhrkamp Verlag, Frankfurt/M erschienen: Paradigmen zu einer Metaphorologie, in Archiv für Begriffsgeschichte 6, 1960, 7-142 (Separatdruck Bonn, 1960). Anthropologische Annäherung an die Aktualität der Rhetorik, in Wirklichkeiten in denen wir leben, Stuttgart, 1981, 104-136. Beobachtungen an Metaphern, in Archiv für Begriffsgeschichte 15, 1971, 161-214 (Sektion IV, «Paradigma, grammatisch», nachgedruckt in Wirklichkeiten 157-162). Ernst Cassirers gedenken bei Entgegennahme des Kuno-Fischer-Preises der Universität Heidelberg 1974, in Wirklichkeiten 163-172. Nachdenklichkeit, Deutsche Akademie für Sprache und Dichtung. Jahrbuch 1980, 57-61 Dankrede anläßlich der Verleihung des Sigmund-FreudPreises für wissenschaftliche Prosa.

5. - Vgl. auch S. 9 (wo «absolut» sich auf die Unübersetzbarkeit der Metapher ins Medium des Begriffs bezieht und nicht speziell auf das Problem der Totalität) sowie die folgende Passage: Die 
absolute Metapher gibt «der durch keine theoretische Untersuchung beantwortbaren Frage nach der Stellung des Menschen im Universum des Seienden einen orientierenden Anhalt» (123).

6. - In diesem gesamten Aufsatz unterscheide ich in der Regel nicht die verschiedenen Arten von Ausdrucks- und Darstellungsformen. Diese wichtigen Unterschiede habe ich hier vernachlässigt, weil ich mich ganz auf das konzentriere, was diesen Formen gemeinsam ist. Metapher, Mythos, Rhetorik und Symbol; Begriff, Vernunft und Wissenschaft; symbolische Formen, Kultur, Systeme des Wissens und «Antworten»: sie alle erfüllen die Funktion, die Neugierde zu sättigen, Bedürfnisse zu befriedigen, «Fragen» zu beantworten, «Leerstellen» zu «besetzen».

7. - Zur Paradigma-Metapher vgl. Anselm Haverkamp. Paradigma Metapher, Metapher Paradigma, in Poetik und Hermeneutik XII (1987) und meine Überlegungen zu Beobachtungen an Metaphern weiter unten.

8. - Meine Zitate stammen, soweit nicht anders vermerkt, aus der Ausgabe von 1966. Vgl. auch die erweiterte und überarbeitete Ausgabe in drei Bänden: Säkularisierung und Selbstbehauptung (1974); Der Prozeß der theoretischen Neugierde (1973); Aspekte der Epochenschwelle (1976).

9. - Blumenbergs Symbolbegriff stellt eine Zuspitzung der distanzschaffenden Funk-tion der Metapher dar: «Der Grenzwert der Metapher fist] das Symbol; hier ist das Andere das ganz Andere, das nichts hergibt als die pure Ersetzbarkeit des Unverfügbaren durch das Verfügbare» (Anthropologische Annäherung 116). Der «Ausblick» - Aufsatz arbeitet die Notwendigkeit dieser Ersetzbarkeit weiter aus: «Das Symbol ist ohnmächtig etwas über seinen Referenzgegenstand mitzuteilen. Dafür steht es für das Nicht-Abbildbare, ohne zu ihm hin zu verhelfen. Es hält die Distanz aufrecht, um zwischen Subjekt und Objekt eine Sphäre ungegenständlicher Korrelate des Denkens, die des symbolisch Darstellbaren, zu konstituieren. Es ist die Möglichkeit der Wirkung der bloßen Idee, der Idee als des Inbegriffs von Möglichkeiten, wie es die des Wertes ist. Oder die des "Seins"» (90 f.).

Dieser Begriff des Symbols steht hinter der Metaphorologie Blumenbergs, und es unterstreicht die Notwendigkeit der Distanz, die Metapher und Mythos schaffen. Kurz gesagt: die Abwesenheit des Absoluten schützt uns vor der Absolutheit des Abwesenden. Vgl. auch Paradigmen 123-142.

10. - Vgl. Aspekte der Epochenschwelle, «Der Begriff der "Umbesetzung" bezeichnet implikativ das Minimum an Identität, das noch in der bewegtesten Bewegung der Geschichte muß aufgefunden oder zumindest vorausgesetzt und gesucht werden können» (17).

11. - Vgl. Blumenbergs Überlegungen zum Thema «Zeit» in Lebenszeit und Weltzeit (1986). Dort wird als terminus a quo die «Lebenswelt» genommen, in der es keine Differenz - oder eigentlich «Indifferenz» - zwischen Lebenszeit und Weltzeit gibt. Dies beschreibt einen anderen Grenzpunkt, der dem des «Mängelwesens» und des «Absolutismus der Wirklichkeit» gegenüber steht: Die Lebenswelt ist der Bereich der Erfahrung, in dem der Mensch der Welt gut angepaßt ist und jedes Bedürfnis seine unmittelbare Befriedigung findet. Wir können uns eine solche Situation als «Vorgeschichte» des Menschen vorstellen; sie liegt dem Absolutismus der Wirklichkeit voraus und beschreibt natürlich auch Aspekte unserer gegenwärtigen Erfahrung. Leider kann eine ausführlichere Diskussion der Bedeutung von Lebenszeit und Weltzeit für die hier behantelten Themen in diesem Aufsatz nicht stattfinden. Eine solche Untersuchung wird die phänomenologischen Aspekte im Werk Blumenbergs ausführlicher behandeln müssen; außerdem wird sie sich zu beschäftigen haben mit jenem Vorhaben, das Blumenberg in der Einleitung zu Wirklichkeiten in denen wir leben angekündigt hat: eine «Phänomenologie der Geschichte» (6) auf den Weg zu bringen, in der Anschauung und Beschreibung ihren Platz neben den Abstraktionen von Begriff und Deduktion einnehmen werden. Zu beachten ist die Bedeutung der Anschauung, wie sie in der unten dargestellten Erörterung des Zuschauers mit zum Ausdruck kommt.

12. - Robert A. Segal, Blumenberg als Theorist of Myth, in Annais of Scholarship 5, 1987, 93. Vgl. auch Robert Wallace, Translateras Introduction, in Work on Myth, Cambridge, 1985, XVI-XIX. Mag sein, daß Blumenberg einer Darstellung des Ursprungs der Inhalte einzelner Mythen ausweicht; er versäumt es indes nicht, seinen eigenen Gedanken über den Ursprung der Funktion des 
Mythos überhaupt «Inhalt» und «Subtanz» zu geben. Die Betonung der Funktion befreit nicht von der Notwendigkeit mit «Inhalten» umzugehen, macht aber in stärkerem Maße bewußt, wie man damit umgeht, macht also aufmerksam auf die eigene Abhängigkeit vom Mythos.

13. - Die Legitimität der Neuzeit weist auch auf ihre eigene metaphorische Basis hin: daß die Rede von der «Umbesetzung» der «Stellen» im «Haushalt» der menschlichen Wissensbedürfnisse ein nur heuristisches Prinzip ist, macht Blumenberg deutlich, indem er selbst diese Ausdrücke immer wieder in Anführungszeichen setzt. Die Paradigmen zu einer Metaphorologie dagegen enthalten keine derartigen Hinweise auf ihre eigene rhetorische Struktur. «Paradigma» und «Daseinsgrund» fungieren da nur untergründig.

14. - Vgl. Die Lesbarkeit der Welt(\9U); Das Lachen der Thrakerin (1987); Höhlenausgänge (1989) und seine umfangreichen Arbeiten über kosmologische Metaphorik, besonders: Die Genesis der kopernikanischen Welt (1975).

15. - Auf die ökumenische Eigenart der Arbeiten Blumenbergs hat mich zuerst Allen Mandelbaum hingewiesen. Als eine andere Einstellung zu den Problemen von Neuzeit und Totalisierung vgl. A Mandelbaum, «Taken from Brindisi»: Vergil in an Other's Otherworld, apeared in: Vergil at 2 000. Ed. John Bernard, New York, 1986, 225-239.

16. - Eine Art der Vergeßlichkeit, die Blumenberg tatsächlich hochschätzt, hat ihren ort vor diesen Fragen, die sie zugleich erzeugte. «Damit das Zurücksinken [des Menschen auf die Stufe seiner Ohnmacht, gleichsam in die archaische Resignation] nicht nur möglich, sondern zum Inbegriff neuer Wünsche wurde, mußte etwas vergessen worden sein. Dieses Vergessen ist die Leistung der Distanz durch «Arbeit am Mythos» selbst. Sie ist die Bedingung für alles, was diesseits des Schreckens, des Absolutismus der Wirk-lichkeit, möglich wurde» (Arbeit am Mythos 15).

Der Gebrauch des Plurals in Ausdrücken wie «Fragen», «Stellen» oder «Probleme» macht deutlich, daß wir selbst in krisenhaften Augenblicken, wenn die Antworten zerfallen, nicht zurückkommen auf oder uns erinnern an jene undifferenzierte Angst, die das einzige und allumfassende «Problem» des Menschen war, welcher sich als erster aufrichtete und den Urwald verließ. Indem die Fragen, die wir stellen, unseren weiten Wahrnehmungshorizont gliedern und ordnen, vermögen sie selbst der Sache des Selbterhaltung dienlich zu sein.

17. - Als eine nützliche Erörterung dieser Ambiguität vgl. Robert Wallace, Hans Blumenberg on Descartes and the Modern Age, in Annals of Scholarship 5, 1987, 37-63.

\section{AUTEUR}

\section{DAVID ADAMS}

Queens College of the City University of New York 